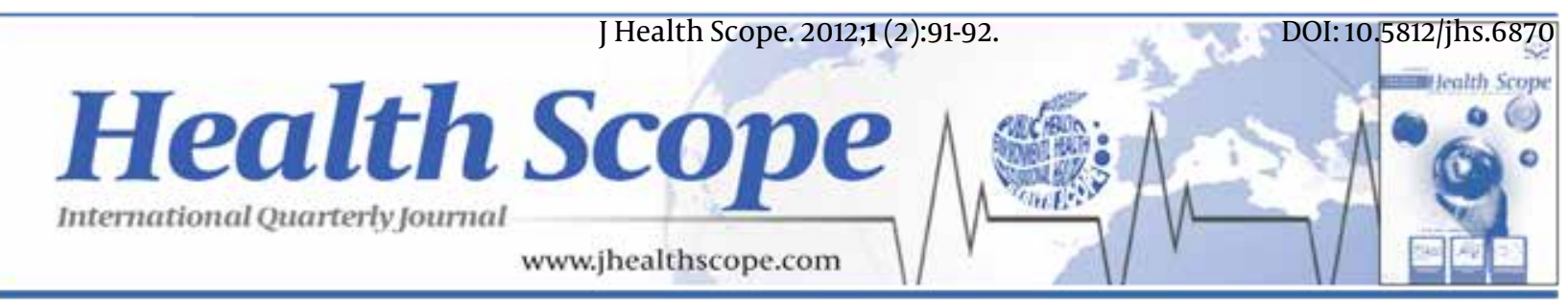

\title{
Short-Term Pre-Pregnancy Health Education May Improve Maternal Nutrition Behavior, but not Nutrition Self-Efficacy
}

\author{
Amanda Rodrigues Amorim Adegboye ${ }^{1^{*}}$ \\ ${ }^{1}$ Research Unit for Dietary Studies, Institute of Preventive Medicine, Copenhagen, Denmark
}

\begin{tabular}{l}
\hline A R T I C L E I N F O \\
\hline Article type: \\
Letter to Editor \\
\hline Article history: \\
Received: 19 Jun 2012 \\
Revised: 23 Jun 2012 \\
Accepted: 11 Jul 2012 \\
\hline Keywords: \\
Pregnancy \\
Health \\
Education
\end{tabular}

\section{Dear Editor,}

Dr. Bastani has presented an interesting randomizedcontrolled trial on the effect of nutritional education on behavioral intention and self-efficacy in women with focus on iron and folic acid intakes in the J Health Scope (1). Iron and folic acid deficiencies during gestation are often associated with poor pregnancy outcomes for both mother and fetus (2). Although pre-conception counseling and nutrition education could be an ideal opportunity to improve maternal daily iron and folic acid intakes before conception, implementation of this form of preventive intervention is still lacking in many non-Western countries, particularly in Iran $(3,4)$. The one-day nutrition workshop, involving 8-12 women from the experimental group $(n=50)$ in each workshop, was preceded by an individualized advise session on healthy lifestyle and benefits of healthy diet. The control group received standard care $(n=49)$. The intervention lead to increased behavioral intention on iron and folic acid intakes among women planning pregnancy, but did not improve self-efficacy (1).
- Please cite this paper as:

Amorim Adegboye AR. Short-Term Pre-Pregnancy Health Education May Improve Maternal Nutrition Behavior, but not Nutrition Self-Efficacy.J Health Scope. 2012; 1 (2): 91-2.

There appears to be confusion on the concept of behavioral intention, self-efficacy and attitudes. It is therefore important to have a clear idea of some definitions before interpreting the findings. Behavioral intention measures a person's relative strength of intention to perform a behavior. Attitude consists of individual's feeling (positive) negative) about the consequences of performing the behavior. Self-efficacy is a person's belief in his/her own competence of enacting a certain behavior to attain a set of goals (5). The findings suggest that in order to improve levels of self-efficacy towards dietary changes with pre-conception women, more intense efforts are likely needed. Providing information on the benefits of healthy dietary habits and raising awareness of problems associated with unhealthy dietary habits may increase the knowledge, but not the willingness and ability to adopt healthy lifestyle. Socio-economic factors, social norms, family attitudes, and fear of failure can be important barriers to change. The current intervention might have benefited from a booster session providing reassurance and constructive feedback. Future intervention may consider

${ }^{*}$ Corresponding author: Amanda RAmorim Adegboye, Institut for Sygdomsforebyggelse/Institute of Preventive Medicine Frederiksberg Hospital. Hovedvejen indgang 5, Nordre Fasanvej 57, 2000 Frederiksberg. Copenhagen, Denmark. Tell:+45-33383772, Fax:+45-33324240, E-mail: AAR@ipm. regionh. dk 
taking into account the participation of family members, such as the husband or partner, and assessment of multiple domains including social support, barriers and encouragement for dietary change. Strengths of the study include the relatively large sample, low attrition rate, and short completion time of the program. The author acknowledges that the generalizations of the findings might be limited due to the peculiar characteristics of the study population. All participants were married; most of them were unemployed, but highly educated, and planning to be pregnant in their first year of marriage and, therefore, more motivated to learn healthy lifestyle.

Future studies will be needed to determine whether improvements in behavioral intention can be sustained over a longer period up to conception and whether self-reported intention to change will ultimately translate into behavior.

\section{Authors' Contribution}

This study has been done completly by auther.

\section{Financial Disclosure}

None declared.

\section{References}

1. Bastani F. The effect of education on nutrition behavioral intention and self-efficacy in women. J Health Scope. 2012;1 (1):12-7.

2. Christian P. Micronutrients, birth weight, and survival. Annu Rev Nutr. 2010;30:83-104.

3. Allaire AD, Cefalo RC. Preconceptional health care model. Eur J Obstet Gynecol Reprod Bio. 1998;78 (2):163-8.

4. Reifsnider E, Gill SL. Nutrition for the childbearing years. J Obstet Gynecol Neonatal Nurs. 2000;29 (1):43-55.

5. Ajzen I, Fishbein M. Understanding attitudes and predicting social behavior. Prentice-Hall; 1980. 